\section{REVISTA}

EDUGACIÓN
Revista Educación

ISSN: 0379-7082

ISSN: 2215-2644

revedu@gmail.com

Universidad de Costa Rica

Costa Rica

\title{
Trayectorias de ingresantes universitarios y estrategias de aprendizaje: sus implicancias en el rendimiento académico
}

Moreno, Jacqueline Elizabet; Chiecher, Analía Claudia; Paoloni, Paola Verónica

Trayectorias de ingresantes universitarios y estrategias de aprendizaje: sus implicancias en el rendimiento académico

Revista Educación, vol. 44, núm. 2, 2020

Universidad de Costa Rica, Costa Rica

Disponible en: http://www.redalyc.org/articulo.oa? $\mathrm{id}=44062184032$

DOI: https://doi.org/10.15517/revedu.v44i2.40055

Esta obra está bajo una Licencia Creative Commons Atribución-NoComercial-SinDerivar 3.0 Internacional. 


\title{
Trayectorias de ingresantes universitarios y estrategias de aprendizaje: sus implicancias en el rendimiento académico
}

\author{
Freshman University Student Approaches to Learning Strategies: Impact on Academic Performance
}

Jacqueline Elizabet Moreno

Universidad Nacional de Rio Cuarto, Argentina

jaqui_rio4@hotmail.com

(iD http://orcid.org/0000-0002-1176-7149

Analia Claudia Chiecher

Universidad Nacional de Rio Cuarto, Argentina

achiecher@hotmail.com

(D) http://orcid.org/0000-0002-5421-6865

\author{
Paola Verónica Paoloni \\ Universidad Nacional de Río Cuarto, Argentina \\ paopaoloni17@hotmail.com \\ (iD http://orcid.org/0000-0002-9384-010X
}

\author{
DOI: https://doi.org/10.15517/revedu.v44i2.40055 \\ Redalyc: http://www.redalyc.org/articulo.oa?id=44062184032
}

Recepción: 30 Enero 2020

Aprobación: 10 Junio 2020

\section{Resumen:}

El estudio describe las estrategias de aprendizaje que utilizaron ingresantes en carreras de ingeniería de la Universidad Nacional de Río Cuarto durante la primera etapa de estudios universitarios y analiza sus relaciones con las trayectorias educativas de logro y de bajo rendimiento. Participaron 86 estudiantes de la cohorte 2018, con trayectorias de rendimiento alto (53\%) y bajo (47\%). Para la recolección de los datos se administró el Cuestionario sobre trayectorias en el primer año universitario que incluye, entre sus secciones, una que evalúa estrategias de aprendizaje. Los resultados de los análisis tanto cuantitativos como cualitativos muestran relaciones positivas entre el uso de estrategias cognitivas complejas, estrategias metacognitivas y de manejo de recursos, por un lado, y el buen desempeño académico por otro. Asimismo, más del $50 \%$ del estudiantado reconoció haber efectuado cambios en sus hábitos de estudio desde el ingreso a la universidad, fundamentalmente en el tiempo dedicado. Para estudiantes de rendimiento alto, el tiempo aparece vinculado a su gestión -es decir, a la planificación, a la organización y a la búsqueda de estrategias efectivas para cumplir con las metas de aprendizaje (55\%)- . Por su parte, los cambios que reconoce el estudiantado de rendimiento bajo están más bien vinculados con el incremento de las horas de dedicación (82\%), lo que no siempre garantiza el alcance de buenos resultados, al menos si no se reflexiona acerca de las estrategias de estudio que permitan un abordaje más profundo de los materiales de estudio. Palabras ClaVE: Trayectoria académica, Estrategias de aprendizaje, Ingreso universitario, Logro, Bajo rendimiento.

\section{Abstract:}

This study describes learning strategies used by engineering students attending the National University of Rio Cuarto (Argentina) during their freshman year. It also addresses their past academic performance and how it impacts their current academic performance. The study group consisted of 86 students from a 2018 cohort, 53\% of whom were high academic performers and the remaining 47\%, low academic performers. The study subjects were asked to complete the survey, Questionnaire on Freshman Year Academic Performance to obtain the required data for the study regarding student academic standing during their first year. The questionnaire included a section that specifically addressed learning strategies employed by students. Findings from this quantitative and qualitative study reveal a positive relationship between the use of certain complex cognitive, metacognitive and resource management strategies and good academic performance. Furthermore, more than $50 \%$ of students stated that they changed their study habits after entering university, particularly, with regards to the time they devoted to studying. According to highachieving students time concurs with better planning, organizing and managing their time, as well as finding the most effective strategies to meet their learning goals (55\%). Low academic performers stated that they altered their habits by incrementing the 
hours spent studying (82\%), which does not always ensure good grades, unless the student examines their own performance and adopts a more profound approach to the course materials.

KEYWORDS: Academic History, Learning Strategies, University Admission, Achievement, Low Performance.

\section{INTRODUCCIÓN}

Aprender a ser estudiante en la universidad es parte de un proceso complejo, que involucra la apropiación no solo de los conocimientos propios de la carrera elegida, sino también el choque con una cultura particular que requiere la incorporación de determinados códigos, costumbres, lenguajes y lugares (Moreno y Chiecher, 2014).

El estudiantado que se incorpora a la vida universitaria sufre el impacto que le generan las dificultades en el desempeño - que se suman al efecto producido por la transición - y que muchas veces se traducen en el desgranamiento de la matrícula. Entre las numerosas dificultades que puede atravesar el alumnado se puede considerar las falencias que puede arrastrar del nivel medio, la falta de preparación del joven en relación con lo que proyecta para su vida futura, la falta de herramientas para enfrentar el estudio universitario al igual que hábitos de estudio e incluso, en muchos casos, la necesidad de incorporarse en simultáneo al ámbito laboral. Por otro lado, frases como: no saben leer, no interpretan las consignas, presentan graves dificultades en la escritura, forman parte de los reclamos de las y los docentes a cargo del primer año y marcan cada vez más una brecha entre un perfil de estudiante ideal y el de estudiante real (Parrino, 2010).

Mientras tanto, los actores de las instituciones se plantean interrogantes en relación con sus responsabilidades: ¿Es el nivel medio el que debe resolver estas problemáticas?, ¿Es responsabilidad del alumnado que está desmotivado y no aprende?, ¿Es la universidad la que debe desarrollar estrategias que brinden algún tipo solución? Si bien no es posible tener todas estas respuestas, las instituciones de nivel superior no pueden permanecer indiferentes ante esta problemática. Por supuesto que el estudiantado tiene responsabilidad en sus procesos de aprender, pues si no adopta una actitud activa y favorable, por más que el contenido sea potencialmente significativo y que el profesorado desarrolle propuestas de enseñanza que efectivamente permitan crear conexiones entre lo que el alumnado ya sabe y las nuevas ideas, difícilmente logre aprender significativamente. Sin embargo, las instituciones educativas juegan un papel primordial en la implementación de estrategias de acción, no solo que aborden la problemática de la retención y deserción, sino que, desde un enfoque más preventivo, reduzcan esta problemática (García, 2015).

Intentando contribuir al conocimiento de esta problemática, el estudio presentado propone describir las estrategias de aprendizaje que utilizaron estudiantes ingresantes en carreras de ingeniería de la Universidad Nacional de Río Cuarto durante la primera etapa del ciclo lectivo y analizar su incidencia en la configuración de distintas trayectorias educativas, especialmente en el logro académico. Se trabajó con la cohorte 2018 y el período considerado para el relevamiento de datos fue el primer cuatrimestre, dado que en el primer año de las carreras de ingeniería las asignaturas son cuatrimestrales.

A continuación, serán dedicados unos apartados a la revisión de algunos aportes teóricos vinculados al estudio de las trayectorias académicas con énfasis en el ingreso universitario y las estrategias de aprendizaje. Luego se avanzará hacia los aspectos metodológicos del estudio y los principales resultados.

\section{La formación entendida como trayectoria.}

El recorrido que desarrolla el estudiantado en el sistema escolar - comparado con la expectativa que supone el diseño de tal sistema -, es lo que se denomina trayectoria académica (Terigi, 2009). Estas trayectorias pueden o no coincidir con lo teóricamente esperado. Terigi (2009) define las trayectorias teóricas o trayectorias ideales, como aquellos recorridos que los sujetos siguen en el sistema en la progresión lineal prevista por los tiempos 
estándar del diseño escolar. Sin embargo, este estereotipo de estudiante ideal o esperado, se confronta con las trayectorias académicas reales, definidas como aquellos recorridos que no siguen exactamente ese cauce previsto, puesto que se ven atravesados por las historias de vida y las situaciones contextuales que a cada sujeto le toca vivir.

En el nivel de Educación Superior no son pocas las personas jóvenes que comienzan una carrera universitaria. Algunos bien parecen seguir la trayectoria que estipula el plan de estudios; sin embargo, existen aquellos que no consiguen un rendimiento aceptable y se retrasan; y otro grupo que poco después de iniciar abandona o decide cambiar de carrera. Considerando la situación particular de las carreras de ingeniería que son las que interesan en este estudio, se encuentra que en Argentina se caracterizan, por lo general, por un decreciente número de inscriptos, un reducido número de graduados, una lentificación en el recorrido de los trayectos curriculares y un abandono marcado de los estudios (Aparicio, 2009; Chiecher, Paoloni y Guebara, 2011; García, Gonzáles y Zanfrillo, 2011; Parrino, 2014). Con diferencias por región y por carrera, la deserción varía entre el $30 \%$ y el $50 \%$ en el país (Panaia, 2013).

El panorama se complejiza aún más en el ingreso universitario. Se registran altas tasas de deserción precoz y temprana; esto es, entre el periodo de inscripción y el momento del ingreso, como así también durante los primeros meses de cursado (Sierra y Hernández, 2016), de hecho, se estima que cerca del 50\% de los ingresantes no llega a completar el primer año (Parrino, 2010).

La propuesta de este trabajo es entonces contribuir al análisis de la problemática a la que se enfrenta la educación universitaria por las demoras en el cursado de la carrera y el marcado abandono, especialmente en el primer año universitario. Se colocará especial énfasis en el conocimiento de las estrategias de aprendizaje empleadas por estudiantes de reciente ingreso en la universidad, con el fin de analizar sus relaciones con las trayectorias que han transitado. Así, algunas de las preguntas que se intentarán responder podrían formularse del siguiente modo: ¿se advierten diferencias en las maneras de abordar el estudio y el aprendizaje entre estudiantes que logran cursar trayectorias exitosas en el primer tramo de estudios universitarios y aquellos que no lo consiguen?, ¿es posible contribuir a potenciar y promover el uso de mejores y más efectivas estrategias de aprendizaje?

Se espera que el conocimiento construido contribuya a ampliar las posibilidades de generar contextos propicios, que potencien el avance exitoso en las trayectorias de formación del alumnado.

\section{Las estrategias de aprendizaje y sus implicancias en el logro académico}

El concepto de estrategias de aprendizaje ha sido ampliamente estudiado en el campo de la Psicología Educacional. Es un constructo complejo y multidimensional y ha sido enriquecido en su estudio, pues en un primer momento el énfasis a la hora de conceptualizarlo estuvo puesto en los aspectos cognitivos y metacognitivos y fue haciéndose mucho más integrador, incluyendo componentes afectivos y motivacionales (García, Sánchez y Risquez, 2016; Vera, Poblete y Díaz, 2019).

Podría decirse que las estrategias de aprendizaje involucran a un conjunto organizado, consciente e intencional de comportamientos, pensamientos, creencias o emociones, que los sujetos ponen en marcha para lograr de manera eficaz un objetivo de aprendizaje en un contexto social determinado. Permiten adquirir nueva información e integrarla al conocimiento y a las habilidades ya existentes.

Para ampliar su definición, los investigadores han identificado diversos rasgos atribuidos a las estrategias de aprendizaje. Son procedimientos que pueden variar en su complejidad; son intencionales porque involucran una acción deliberada del sujeto, con el fin de lograr un objetivo; son esenciales porque debido a sus características permiten lograr experiencias en un determinado conocimiento; son facilitadoras, puesto que están asociadas a las mejoras en los desempeños académicos y por último, requieren un esfuerzo deliberado y voluntad de quien las emplea (Donolo, Chiecher, Paoloni y Rinaudo, 2008). 
Pintrich, Smith, García y McKeachie (1991) y Pintrich y García (1993) desarrollan una descripción muy detallada acerca de los componentes cognitivos implicados en los aprendizajes académicos y distinguen tres tipos de estrategias: cognitivas, metacognitivas y las estrategias de regulación de recursos.

Entre las estrategias cognitivas se encuentran las estrategias de repaso, las estrategias de elaboración y organización de la información y el pensamiento crítico. Las estrategias de repaso son las más simples y generales, puesto que solo permiten un procesamiento superficial del contenido a aprender. En este sentido, solo estarían implicadas en la atención y codificación de la información. Las estrategias de elaboración y organización, en cambio, requieren de un proceso más complejo que posibilita construir conexiones internas entre conceptos, integrar la nueva información con el conocimiento previo posibilitando procesamientos más profundos de los materiales de estudio. Finalmente, desde la perspectiva de los autores mencionados, el pensamiento crítico también es considerado una estrategia cognitiva, pues es el que posibilita al alumnado pensar de manera reflexiva sobre el contenido que está aprendiendo, comprenderlo de una manera más profunda, ser capaz de interpretarlo y dar su propio punto de vista.

Las estrategias metacognitivas se vinculan con el conocimiento y control de sí mismo, de la tarea y de las estrategias que se utilizan para resolverla. Los autores distinguen tres procesos generales: planeamiento, control y regulación. El planeamiento es anterior a la ejecución de la tarea e involucra el conocimiento previo y las actividades que dirigirán y organizarán la conducta del estudiantado a lo largo del desarrollo de la tarea. Las estrategias de control, se utilizan durante el proceso de ejecución de la tarea e implican evaluar los niveles de atención, ajustar el tiempo y esfuerzo, cuestionarse o formular preguntas, evaluar la capacidad para seguir el plan trazado y su eficacia. Por fin, las estrategias de regulación refieren al continuo ajuste de las acciones cognitivas que se desarrollan en función del control previo, en otras palabras, son las encargadas de verificar el proceso de aprendizaje y pueden llevarse a cabo durante y al final de la tarea.

Por último, las estrategias de regulación de recursos incluyen la organización del tiempo y del ambiente de estudio, la regulación del esfuerzo, el aprendizaje con pares y la búsqueda de ayuda. Tal y como su nombre lo indica, la organización del tiempo y del ambiente de estudio involucran comportamientos estratégicos que ayudan a ajustar ciertos factores del contexto, como el hecho de programar y planear los momentos de estudio y seleccionar los lugares de trabajo más favorables, a fin de alcanzar las metas propuestas.

La regulación del esfuerzo está muy relacionada con el compromiso asumido respecto de las actividades o tareas solicitadas como parte del proceso de formación profesional porque tiene que ver con la habilidad del cada estudiante para persistir en estas, a pesar de las distracciones o la falta de interés. El aprendizaje con pares está vinculado a la disposición a trabajar con otros y de manera colaborativa, muy relacionado con la estrategia de búsqueda de ayuda ya que esta modalidad de trabajo, habilita la posibilidad de plantear las dificultades a otros compañeros o a un profesor (Pintrich et al., 1991; Pintrich y García, 1993).

Conforme lo expresado cabe preguntarse: ¿Cuáles son las estrategias que frecuentemente emplean los ingresantes universitarios para resolver sus tareas académicas o para estudiar de cara a un examen?, ¿Son similares o distintas entre estudiantes cuyas trayectorias y rendimiento difieren? Desde el ingreso a la universidad, ¿Ha cambiado el estudiantado sus formas de estudiar intentando ajustarse o adaptarse al nuevo contexto?, ¿O continúan usando las estrategias y modalidades que consolidaron en el nivel secundario? ¿Existen relaciones entre el uso que efectúa el alumnado de determinadas estrategias y la configuración de trayectorias de logro o de bajo rendimiento?

\section{Metodología}

El estudio que se presenta es de tipo no experimental, transeccional-descriptivo (Hernández, Fernández y Baptista, 2014). Propone indagar sobre las estrategias de aprendizaje que utilizaron estudiantes ingresantes en carreras de ingeniería de la Universidad Nacional de Río Cuarto durante la primera etapa del ciclo lectivo 2018 y analizar posibles relaciones con la configuración de distintas trayectorias académicas (de logro y de bajo rendimiento).

Sujetos participantes del estudio 
El estudio involucra a estudiantes que en el año 2018 se inscribieron para cursar alguna de las carreras de ingeniería que ofrece la Universidad Nacional de Río Cuarto ${ }^{[1]}$. La cohorte en su conjunto estuvo compuesta por 218 aspirantes, cuyo rendimiento fue monitoreado al finalizar el primer cuatrimestre de cursado. Del total, 61 sujetos (tan solo el $28 \%$ del total) mostraron trayectorias de logro o de rendimiento alto al finalizar el cuatrimestre, es decir, lograron regularizar todas las asignaturas previstas para ese periodo; 76 sujetos (35\%) informarontrayectorias de rendimiento medio, puesto que habían regularizado solo algunas de las materias según el plan de estudio para el período considerado. Por fin, los 81 sujetos restantes (37\%) mostraron trayectorias de bajo rendimiento, es decir, no lograron la regularidad en ninguna de las asignaturas del plan de estudios durante la primera etapa.

Los datos para este estudio fueron recogidos mediante la administración de un cuestionario el cual, aunque se envió a cada estudiante, fue respondido por un total de 135: 46 de 61 con trayectorias de rendimiento alto (75\%), 49 de 76 estudiantes con rendimiento medio (64\%) y 40 de 81 con trayectorias de bajo rendimiento $(49 \%)^{[2]}$.

A los fines del análisis que se pretenden desarrollar en este estudio, se tomarán en consideración las respuestas proporcionadas por los grupos extremos de alto rendimiento (46 estudiantes) y de bajo rendimiento (40 estudiantes). En ambos grupos los jóvenes encuestados tenían en su mayoría 18 o 19 años. En el caso de estudiantes de alto rendimiento el 67\% (31) eran varones y el 33\% (15) eran mujeres. Mientras que en el alumnado de bajo rendimiento el $82 \%$ (34) son varones y el 18\% (6) restante, mujeres.

\section{Instrumento de recolección de datos}

Para obtener datos relacionados con las estrategias para aprender que emplearon los ingresantes universitarios durante el primer cuatrimestre, se administró el Cuestionario sobre trayectorias en el primer año universitario. Este instrumento ha sido validado resultando, conforme las opiniones de jueces expertos, apropiado para conocer algunas de las variables personales y contextuales que inciden en las trayectorias estudiantiles (Bossolasco, Chiecher y Dos Santos, 2019). Está diseñado en formato online ${ }^{[3]}$ y cuenta con dos versiones que se adaptan de acuerdo al grupo al que se administran: 1) la versión 1 está destinada a estudiantes con trayectorias de logro o de alto rendimiento, es decir, quienes han logrado regularizar todas las asignaturas del período considerado; 2) la versión 2, está destinada a estudiantes con trayectorias de rendimiento medio y bajo, es decir, a quienes regularizaron solo alguna/s o bien ninguna de las materias previstas en el plan de estudio para el período de tiempo considerado.

El cuestionario está estructurado en 10 secciones orientadas a evaluar distintas dimensiones. En este artículo se presentan los resultados correspondientes a la sección VII, que evalúa la dimensión vinculada con las estrategias de aprendizaje. Incluye 12 ítems vinculados al uso de diferentes estrategias de aprendizaje y preguntas abiertas, de respuesta libre. Los 12 ítems que indagan sobre las estrategias de aprendizaje toman como referencia la clasificación de Pintrich et al. (1991):

${ }^{*}$ Estrategias cognitivas (repaso, elaboración y organización y pensamiento crítico).

*Estrategias metacognitivas (planeamiento, control y regulación).

${ }^{*}$ Estrategias de manejo de recursos (regulación del esfuerzo, manejo del tiempo y ambiente, aprendizaje con pares y búsqueda de ayuda).

A continuación, en la Tabla 1, se describen cada uno de los ítems correspondientes a la dimensión de las estrategias para aprender ${ }^{[4]}$ :

Tabla 1

Ítems evaluados en el Cuestionario sobre trayectorias en el primer año universitario, correspondientes a la sección VII, acerca de las estrategias para aprender 
TABLA 1

\begin{tabular}{|c|c|}
\hline Estrategias & Ítems evaluados \\
\hline \multirow{4}{*}{ Cognitivas } & $\begin{array}{l}\text { 1. Cuando estudio para preparar un } \\
\text { examen repaso los contenidos varias veces } \\
\text { y trato de repetirlos para mí mismo o para } \\
\text { otros así los memorizo mejor. }\end{array}$ \\
\hline & $\begin{array}{l}2 . \quad \text { Cuando estudio para un examen } \\
\text { realizo lecturas de los materiales, subrayo } \\
\text { ideas principales y hago resúmenes para } \\
\text { sistematizar la información más } \\
\text { importante. }\end{array}$ \\
\hline & $\begin{array}{l}\text { 3. Cuando estudio para un examen } \\
\text { realizo cuadros, diagramas o gráficos a } \\
\text { partir de la información que he leído. Eso } \\
\text { me ayuda a sistematizar y memorizar. }\end{array}$ \\
\hline & $\begin{array}{l}\text { 4. Siempre que estudio trato de pensar } \\
\text { cuál es mi punto de vista a partir de las } \\
\text { ideas que presentan los materiales. }\end{array}$ \\
\hline \multirow[t]{2}{*}{ Metacognitivas } & $\begin{array}{l}\text { 5. Cuando estudio trato de identificar } \\
\text { conceptos que no comprendo o ejercicios } \\
\text { que no me salen y asi poder trabajar sobre } \\
\text { ellos. }\end{array}$ \\
\hline & $\begin{array}{l}\text { 6. Cuando estudio para un examen me } \\
\text { pongo metas, fijo plazos y voy tratando de } \\
\text { Cumplirlos. }\end{array}$ \\
\hline \multirow{6}{*}{$\begin{array}{l}\text { Manejo de } \\
\text { recursos }\end{array}$} & $\begin{array}{l}\text { 7. Cuando estudio para un examen, } \\
\text { intento persistir y sostener el esfuerzo aun } \\
\text { cuando esté cansado o aburrido. }\end{array}$ \\
\hline & $\begin{array}{l}\text { 8. Cuando estudio para un examen trato } \\
\text { de fijar un horario y planificar el tiempo } \\
\text { disponible. }\end{array}$ \\
\hline & $\begin{array}{l}\text { 9. Cuando estudio para un examen elijo } \\
\text { un ambiente libre de distracciones. }\end{array}$ \\
\hline & $\begin{array}{l}\text { 10. Cuando estudio para preparar un } \\
\text { examen generalmente lo hago con un grupo } \\
\text { de compañeros. }\end{array}$ \\
\hline & $\begin{array}{l}\text { 11. Cuando me estoy preparando para un } \\
\text { examen y no comprendo algún concepto, } \\
\text { tema o no me sale un ejercicio, le pido } \\
\text { ayuda al profesor. }\end{array}$ \\
\hline & $\begin{array}{l}\text { 12. Cuando me preparo para un examen y } \\
\text { no comprendo algún concepto, tema o no } \\
\text { me sale un ejercicio, le pido ayuda a mis } \\
\text { compañeros. }\end{array}$ \\
\hline
\end{tabular}

Fuente: Elaboración propia.

Se solicitó a cada estudiante leer con detenimiento cada uno de los ítems propuestos y responder en una escala de 1 a 7 cuán de acuerdo estaban con las afirmaciones vertidas en ellos.

Los valores en la escala de 1 a 7 son interpretados de acuerdo a las siguientes consideraciones:

(1 - 2) Realiza un uso bajo o nulo de la estrategia mencionada.

(3 - 5) Realiza un uso intermedio u ocasional de la estrategia mencionada

(6-7) Realiza un uso alto ofrecuente de la estrategia.

Por último, y para profundizar en el conocimiento de las estrategias que utilizan los recién llegados a la universidad, se les invitó a responder de manera libre a dos consignas que también forman parte de la sección VII del cuestionario: 1) Describir detalladamente el modo en que se prepara para rendir un examen (parcial o final) de la carrera y 2) precisar si desde el momento en que ingresó a la universidad hasta el momento del relevamiento cambió su forma de estudiar. En el caso que la respuesta fuera positiva, debían describir qué aspectos habían decidido modificar en cuanto a la manera de estudiar. 


\section{Análisis de los datos}

Tal y como se anticipó en apartados anteriores, para el análisis de los datos recogidos se considerarán, del total de ingresantes encuestados, los dos grupos extremos de estudiantes ( $N=86): 1$ ) 46 estudiantes con trayectorias de logro o de alto rendimiento, es decir, estudiantes que regularizaron todas las asignaturas del plan de estudios correspondientes al primer cuatrimestre y 2) 40 estudiantes con trayectorias de bajo rendimiento, esto es, estudiantes que no regularizaron ninguna de las asignaturas cursadas durante el primer cuatrimestre.

Por otro lado, los resultados serán presentados en dos partes; la primera, que incluye los resultados obtenidos de las respuestas a 12 ítems correspondientes al uso de estrategias de aprendizaje, cuyos análisis serán cuantitativos ya que fueron respondidos en base a la escala Likert.

La segunda parte, presenta el análisis de las respuestas a las preguntas abiertas que dieron los ingresantes en relación con: 1) el modo en que se preparan para rendir un examen y 2) si cambiaron o no sus estrategias de estudio desde el momento que comenzaron la universidad. Estos datos se analizaron inductivamente, procediendo a la construcción y definición de categorías emergentes de las respuestas escritas por el estudiantado.

\section{Resultados}

\section{Primera parte: Análisis cuantitativo de respuestas a ítems basados en escala Likert.}

A continuación, se presentan los resultados obtenidos al analizar comparativamente las respuestas de los sujetos de alto y bajo rendimiento a los 12 ítems del cuestionario basados en escala Likert.

Tabla 2

Uso de estrategias cognitivas, metacognitivas y de manejo de recursos. Datos para 86 ingresantes 
TABLA 2

\begin{tabular}{|c|c|c|c|}
\hline Estrategias cognitivas & Frecuencia de uso de estrategias & Grupo de alto rendimiento & Grupo de bajo rendimiento \\
\hline \multirow[t]{3}{*}{ Estrategias de repaso } & Aito & $45,7 \%$ & $45 \%$ \\
\hline & Intermedio & $37 \%$ & $47,5 \%$ \\
\hline & Bajo & $17,4 \%$ & $7,5 \%$ \\
\hline \multirow[t]{3}{*}{ Estrategias de elaboración de la información } & Aito & $71,7 \%$ & $55 \%$ \\
\hline & Intermedio & $26,1 \%$ & $42,5 \%$ \\
\hline & Bajo & $2,2 \%$ & $2,5 \%$ \\
\hline \multirow[t]{3}{*}{ Estrategias de organización de la información } & Alto & $26,1 \%$ & $20 \%$ \\
\hline & Intermedio & $54,3 \%$ & $57,5 \%$ \\
\hline & Bajo & $19,6 \%$ & $22,5 \%$ \\
\hline \multirow[t]{3}{*}{ Pensamiento Crítico } & Alto & $34,8 \%$ & $40 \%$ \\
\hline & Intermedio & $56,5 \%$ & $52,5 \%$ \\
\hline & Bajo & $8,7 \%$ & $7,5 \%$ \\
\hline \multicolumn{4}{|l|}{ Estrategias metacognitivas } \\
\hline \multirow[t]{3}{*}{ Estrategias vinculadas a la identificar dificultades } & Alto & $82,6 \%$ & $55 \%$ \\
\hline & Intermedio & $17,4 \%$ & $40 \%$ \\
\hline & Bajo & --- & $5 \%$ \\
\hline \multirow[t]{3}{*}{ Estrategias de planeamiento, control y evaluación } & Alto & $54,3 \%$ & $35 \%$ \\
\hline & Intermedio & $34,8 \%$ & $45 \%$ \\
\hline & Bajo & $10,9 \%$ & $20 \%$ \\
\hline \multicolumn{4}{|l|}{ Estrategias de manejo de recursos } \\
\hline \multirow[t]{3}{*}{ Regulación del esfuerzo } & Aito & $50 \%$ & $37,5 \%$ \\
\hline & Intermedio & $34,8 \%$ & $52,5 \%$ \\
\hline & Bajo & $15,2 \%$ & $10 \%$ \\
\hline \multirow[t]{3}{*}{ Organización del tiempo } & Alto & $54,3 \%$ & $37,5 \%$ \\
\hline & Intermedio & $32,6 \%$ & $50 \%$ \\
\hline & Bajo & $13 \%$ & $12,5 \%$ \\
\hline \multirow[t]{3}{*}{ Organización del ambiente } & Alto & $63 \%$ & $57,5 \%$ \\
\hline & Intermedio & $32,6 \%$ & $35 \%$ \\
\hline & Bajo & $4,3 \%$ & $7,5 \%$ \\
\hline \multirow{3}{*}{ Aprendizaje con pares } & Alto & $23,9 \%$ & $25 \%$ \\
\hline & Intermedio & $52,2 \%$ & $52,5 \%$ \\
\hline & Bajo & $23,9 \%$ & $22,5 \%$ \\
\hline \multirow[t]{3}{*}{ Búsqueda de ayuda: profesores } & Alto & $71,7 \%$ & $30 \%$ \\
\hline & Intermedio & $26,1 \%$ & $65 \%$ \\
\hline & Bajo & $2,2 \%$ & $5 \%$ \\
\hline \multirow[t]{3}{*}{ Búsqueda de ayuda: compañeros } & Alto & $67,4 \%$ & $57,5 \%$ \\
\hline & Intermedio & $28,3 \%$ & $35 \%$ \\
\hline & Bajo & $4,3 \%$ & $7,5 \%$ \\
\hline
\end{tabular}

Fuente: Elaboración propia.

\section{Acerca de las estrategias cognitivas}

Respecto de las estrategias de repaso se puede observar que los porcentajes se mantienen bastante equiparados en los dos grupos de estudiantes analizados. En efecto, los porcentajes más altos corresponden al uso frecuente e intermedio de la estrategia referida. También es de destacar que, entre estudiantes de alto rendimiento, un mayor porcentaje ( $17,4 \%$ frente a $7,5 \%$ que informaron estudiantes de bajo rendimiento) dijo no recurrir frecuentemente a esta estrategia, lo cual es esperable dado que, como previamente dijimos, no es una estrategia que se asocie con procesamientos profundos, sino que más bien, conduce a un procesamiento superficial de los materiales de estudio. Sin embargo, también suelen utilizarse cuando los materiales son muy complejos y requieren aprendizajes basados en la asociación (Pozo, 1990), lo cual podría explicar los elevados porcentajes de empleo tanto en el alumnado de bajo, como aquellos de alto rendimiento.

Las estrategias de elaboración - al igual que las de organización - involucran procesamientos profundos de la información y facilitan la construcción de significados a partir de la conexión interna entre ideas y en la literatura están vinculados con aprendizajes significativos y mejores desempeños académicos (Pozo, 1990). Ambas estrategias requieren de una participación mucho más activa del estudiantado, tanto en el proceso, como en el producto de la tarea - aspecto difícil de observar en estrategias de repaso -.

Los resultados de este estudio muestran cierta inclinación de estudiantes de alto rendimiento por el uso de estrategias de elaboración en mayor medida que los de bajo rendimiento. Las respuestas muestran que el 71,7\% 
de estudiantes con trayectorias de alto rendimiento hacen uso alto o frecuente de este tipo de estrategias mientras que entre estudiantes de rendimiento académico bajo solo el $55 \%$ apela con frecuencia a su uso.

No obstante, los resultados obtenidos no muestran diferencias en el uso de estrategias de organización de la información. De hecho, el mayor porcentaje corresponde al uso intermedio u ocasional de la estrategia: 54,3\% en el caso del alumnado con trayectoria exitosas y asciende a un $57,5 \%$ de estudiantes con trayectorias de bajo rendimiento. Solo el $26,1 \%$ de estudiantes con rendimiento alto y un $20 \%$ del alumnado con rendimiento bajo mencionaron utilizarla frecuentemente.

Finalmente, en cuanto al pensamiento crítico, por ser una estrategia que requiere un rol activo del/ la alumno/a, ya sea por pensar de manera reflexiva o por intentar interpretar de una manera distinta el contenido a aprender, podría considerarse que es más empleada por aquellos(as) estudiantes cuyos desempeños académicos son más exitosos. En el caso de los ingresantes encuestados, el mayor porcentaje de ambos grupos corresponde al uso intermedio u ocasional de esta estrategia; $56,5 \%$ en el caso de estudiantes con rendimiento alto y $52,5 \%$ en el caso de estudiantes con rendimiento bajo. Por otro lado, se encuentra que el grupo correspondiente a las trayectorias de bajo rendimiento supera con un $40 \%$ (frente a un 34,8\%) al grupo con rendimiento alto, indicando el uso alto o frecuente de la estrategia en cuestión.

\section{Acerca de las estrategias metacognitivas}

Se pueden observar diferencias entre los grupos analizados en relación con el uso de estrategias metacognitivas, vinculadas de manera positiva con aquellos(as) estudiantes que tienen mejor desempeño académico.

El ítem que refiere a estrategias vinculadas a identificar dificultades, sugiere la capacidad de estudiantes de reconocer sus propias dificultades frente a una tarea o a un material de estudio. El 82,6\% de estudiantes con alto rendimiento reconocieron explotar con alta frecuencia esta habilidad, mientras que del grupo con bajo rendimiento lo hace un $55 \%$ frecuentemente y el $40 \%$ lo hace, pero ocasionalmente.

En cuanto a estrategias de planeamiento, control y evaluación, se pueden observar aún mayores diferencias entre los dos grupos analizados. En el caso de estudiantes con trayectorias de bajo rendimiento el mayor porcentaje usa ocasionalmente la estrategia en cuestión, seguido por un 35\% que indicó hacerlo con mayor frecuencia. Por el contrario, en el caso del alumnado con rendimiento alto, se encuentra que el mayor porcentaje, es decir más del 50\%, indicó emplear frecuentemente estrategias que les permitan planificar, controlar y evaluar sus procesos de aprendizaje.

Las estrategias metacognitivas en la bibliografía, están vinculadas con el buen rendimiento académico. El monitoreo y la regulación del propio desempeño para el logro de metas personales y educacionales, promueve en el estudiantado la capacidad de construir aprendizajes significativos, pues lo que caracteriza principalmente a este alumnado es que tiene conocimiento de las estrategias efectivas para él, sabe cómo evaluarlas y ajustar aquellas estrategias inadecuadas para el cumplimiento de una tarea. De esta manera, enfocan sus aprendizajes de manera más reflexiva y comprometida, obteniendo así mejores logros académicos (Garello y Rinaudo, 2012).

\section{Acerca de las estrategias de manejo de recursos}

Las estrategias de regulación del esfuerzo son de carácter volitivo y hacen referencia a la capacidad de cada estudiante para mantener el esfuerzo y la concentración en la tarea evitando las distracciones internas o externas, ante tareas poco interesantes o difíciles. En otras palabras, tiene que ver con el conjunto de estrategias que le permiten mantener la decisión de seguir centrado en la tarea cuando aparecen obstáculos, ya sea en la misma situación de aprendizaje o en la motivación (Broc, 2011). 
Los resultados de este estudio muestran que el estudiantado de alto rendimiento es algo más hábil en este aspecto. Tal y como como puede observarse en la Tabla 2, el 50\% del alumnado con rendimiento alto expresó emplear estrategias de regulación del esfuerzo de manera frecuente. En cambio, en el grupo de rendimiento bajo fue mayoritario el porcentaje de quienes usan ocasionalmente la estrategia (52,5\%).

En relación con las estrategias de manejo del tiempo de estudio, el 54,3\% del estudiantado con trayectorias de alto rendimiento expresó emplear frecuentemente estrategias vinculadas a la organización del tiempo de estudio, mientras que el $50 \%$ con trayectorias de bajo rendimiento lo hace, pero de manera ocasional.

En cambio, con respecto a estrategias de manejo del ambiente, las diferencias entre los grupos son menores, de hecho, tanto en el grupo de trayectorias de alto como de bajo rendimiento, los mayores porcentajes (63\% y $57,5 \%$ respectivamente), corresponden al uso alto o frecuente de este tipo de estrategias.

Se puede apreciar entonces que las estrategias que refieren a una planificación y organización del tiempo son consideradas en menor medida por el alumnado de bajo rendimiento. Los resultados demuestran que hay mayor preferencia en la selección de un buen lugar de trabajo al momento de estudiar o ejecutar tareas académicas. Sin embargo, esto último no tiene tanta implicancia en la regulación y control de los propios procesos de aprendizaje como el manejo de tiempo.

En cuanto al aprendizaje con pares claramente no se observan diferencias entre los grupos analizados. En efecto, el mayor porcentaje representa el uso intermedio de la estrategia en cuestión; 52,2\% en estudiantes de rendimiento alto y $52,5 \%$ en estudiantes con rendimiento bajo.

Se han destacado los beneficios del trabajo en grupo como estrategia sistemática, no solo por el valor pedagógico que poseen para el desempeño académico y para la regulación de los aprendizajes, sino también para la formación de competencias necesarias para su desarrollo profesional (Garzuzi, 2015). Sin embargo, generar un clima de confianza e igualitario no es tan sencillo cuando recién se ingresa a la universidad, porque seguramente es muy poco lo que conocen de sus propios compañeros de curso y de las formas de trabajo. Esto podría explicar los porcentajes de empleo intermedios y el aumento de los porcentajes que refieren a los usos bajos u ocasionales, si se compara con otras estrategias.

En cuanto a la búsqueda de ayuda, de acuerdo con los resultados obtenidos, se puede observar que el alumnado con mejor rendimiento parece más proclive a solicitar ayuda a su docente, antes que a sus compañeros. Mientras que el 71,7\% manifestó que siempre pide ayuda al profesorado, el $67,4 \%$ expresó que siempre solicita la ayuda de sus compañeros.

Con el grupo de estudiantes de rendimiento bajo, sucede lo contrario. Se muestran más predispuestos a solicitar ayuda de los compañeros, antes que a sus docentes. El 57,5\% expresó que cuando requiere de alguna ayuda, frecuentemente busca a sus compañeros, mientras que el $30 \%$ prefiere consultar al profesorado.

Los resultados de la prueba $t$ con muestras independientes permiten apreciar que, en términos generales, las medias para cada ítem asumen valores superiores en el grupo de estudiantes de alto rendimiento (excepto en repaso, que es esperable, y en pensamiento crítico). No obstante, en coincidencia con los resultados que se han presentado hasta aquí, las diferencias son estadísticamente significativas para tres de los ítems relativos a: 1) uso de estrategias de elaboración ( $t=1,978$; gl 84; sig. 052), 2) uso de estrategias metacognitivas de identificación de conceptos o ejercicios complejos para trabajar sobre ellos ( $t=2,409$; gl 84; sig. .018) y, 3) búsqueda de ayuda dirigida al profesor ( $\mathrm{t}=3,844 \mathrm{gl} 84$; sig. .000). En todos los casos, las medias fueron más altas en el grupo de estudiantes de alto rendimiento. 


\section{Segunda parte: Análisis cualitativo a partir de las respuestas escritas del grupo de estudiantes}

En esta sección se presentan los resultados del análisis cualitativo, elaborado sobre respuestas de los sujetos a preguntas abiertas, las cuales complementan y amplían la perspectiva respecto de la información proporcionada hasta aquí.

\section{Acerca del modo en que se preparan para rendir un examen}

A continuación, se describen las categorías de respuesta enunciadas por el grupo de estudiantes, las cuales aluden al modo en que se preparan para rendir un examen. Las categorías fueron construidas inductivamente a partir de las recurrencias en las respuestas ${ }^{[5]}$. Los resultados se presentan diferenciados por trayectoria de rendimiento (alto y bajo).

Estudiantes con trayectorias de rendimiento alto

El Gráfico 1 resume las categorías elaboradas a partir de las respuestas del grupo de estudiantes de alto rendimiento en las entrevistas.

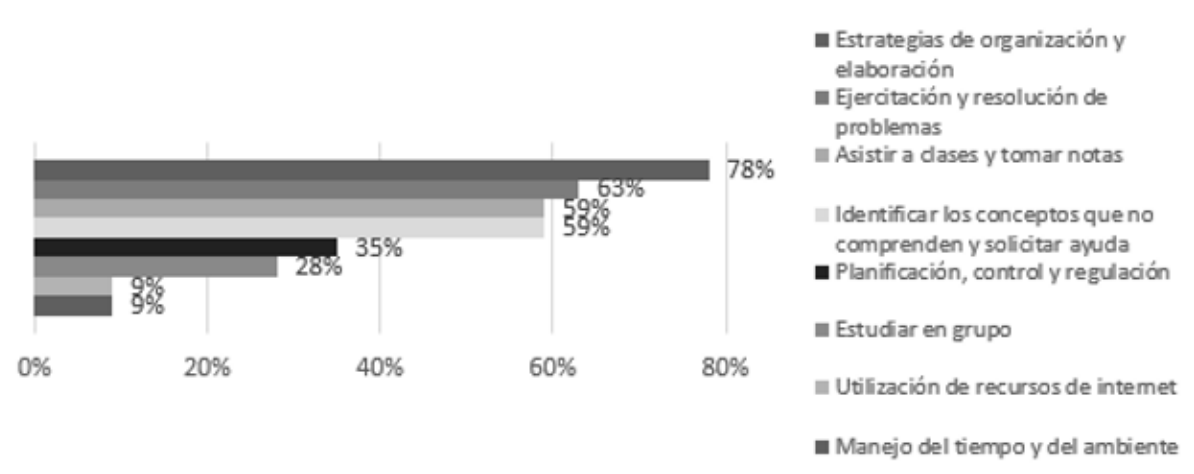

GRÁFICO 1

Acerca del modo en el que se preparan estudiantes con trayectorias de rendimiento alto para rendir un examen $(\mathrm{N}=46)$

Fuente: Elaboración propia.

Estrategias de elaboración y organización (78\%): la mayoría de estudiantes de alto rendimiento manifestó que comprender, ser analíticos y relacionar es la manera más efectiva de estudiar. Para llevar a cabo este proceso, optan por estrategias de elaboración y organización de la información, como la ejecución de resúmenes, síntesis, cuadros que les permitan establecer relaciones, buscan ejemplos, efectúan lecturas profundas de los materiales de estudio, elaboran preguntas a los textos, identifican ideas principales y secundarias y se preocupan por tener en claro los conceptos que luego aplicarán en la resolución de diversas situaciones problemáticas que les propone el o la docente. A continuación, se presenta un fragmento que ilustra las respuestas del grupo de estudiantes.

Agarro las notas que he tomado en clases, y empiezo a hacer resúmenes, leo el material o libro que tengo que estudiar y subrayo lo más importante, lo comprendo y luego escribo en una hoja lo que me quedó en claro con mis palabras (F.F., comunicación personal, agosto 2018)

Ejercitación y resolución de problemas (63\%). Este grupo de estudiantes considera parte indispensable en sus hábitos de estudio resolver diferentes tipos de ejercicios prácticos que proporciona el/a profesor/a en clase o los que encuentren en los materiales de estudio, aprender fórmulas e identificar cuál es la más pertinente según la situación problemática que deben resolver. 
Para los parciales de fisica me centraba en aprenderme las fórmulas y practicar mucho los ejercicios encontrados en internet o en los apuntes. Cada vez que realizaba un ejercicio copiaba todas las fórmulas y luego elegía cuál era la que necesitaba para realizar el ejercicio (E. S., comunicación personal, agosto 2018).

Asistir a clases y ser muy bueno tomando notas (59\%). El estudiantado considera la asistencia regular a clases como parte importante de su preparación ante los exámenes y como una variable fundamental en la aprobación de las asignaturas.

Como siempre, trato de ir a todas las clases y tomo nota de todo, me sirve a la hora de poder resolver un ejercicio sola, ya que aún no formé un grupo de estudio (L. B., comunicación personal, agosto 2018).

Identificar los conceptos que no comprenden y solicitar ayuda (59\%). El pedido de ayuda tanto a compañeros, como a las y los profesores durante las clases y horarios de consulta, fue mencionado por más de la mitad del estudiantado de alto rendimiento como una estrategia a la cual recurren cuando se preparan para un examen.

Tomo apuntes en clases y asisto a clases consultas o le pregunto a compañeros mis dudas para poder comprender mejor (A. G., comunicación personal, agosto 2018).

Planificación, control y regulación (35\%). Para llevarla a cabo no solo consideran el tiempo que les queda antes del examen, sino que se preocupan por conocer qué es lo que deben saber. Esto les permite fijarse metas a corto plazo para ir controlando sus logros, identificar aquello que no comprenden y fijarse horarios de estudio que tratan de cumplir siempre para ir al día con los contenidos que ven en clases.

Yo me propongo estudiar hasta cierta parte en un dia pensando en el tiempo disponible que tengo y eso que me propongo lo tengo que cumplir, son como metas a corto plazo que me planteo cada día para que después no se me acumule todo junto (C. R., comunicación personal, agosto).

Estudiar en grupo (28\%). Algo menos de un tercio del alumnado expresó haber logrado constituir un grupo de estudio. Aunque solo para resolver ejercicios y situaciones problemáticas. Los conceptos teóricos en su mayoría prefieren analizarlos de manera individual y recurren a los pares para evacuar dudas, si las hubiere.

Me reúno con compañeros para reforzar lo aprendido y abordarlo desde distintos puntos de vista. Mientras estoy solo únicamente repaso y en el examen trabajo concentrado y seguro (M. B., comunicación personal, agosto).

Utilización de recursos de internet (9\%). Un grupo minoritario (9\%) utiliza recursos de internet, (páginas web, Youtube) para ampliar sus materiales de estudio, buscar ejemplos, ejercicios o comprender conceptos, cuando no hay un otro en el momento que pueda ayudarlos.

Primero leo y subrayo todo el material, luego busco en internet conceptos que no comprendo o que no aparecen en el material (L. C., comunicación personal, agosto 2018).

Manejo del ambiente (9\%). Aunque no son muchos, algunos(as) estudiantes destacan explícitamente que para acompañar sus hábitos de estudio es fundamental el manejo del ambiente donde se lleva a cabo este. Buena música o un lugar tranquilo y silencioso, pequeños recreos que se regalan y contar al menos con alguna actividad que brinde un momento de relajación previo a ir a rendir.

Busco un lugar tranquilo de mi casa donde puedo concentrarme (F.F., comunicación personal, agosto 2018).

Estudiantes con trayectorias de rendimiento bajo

El Gráfico 2 expone las categorías elaboradas a partir de las respuestas del grupo de estudiantes de rendimiento bajo en las entrevistas. 


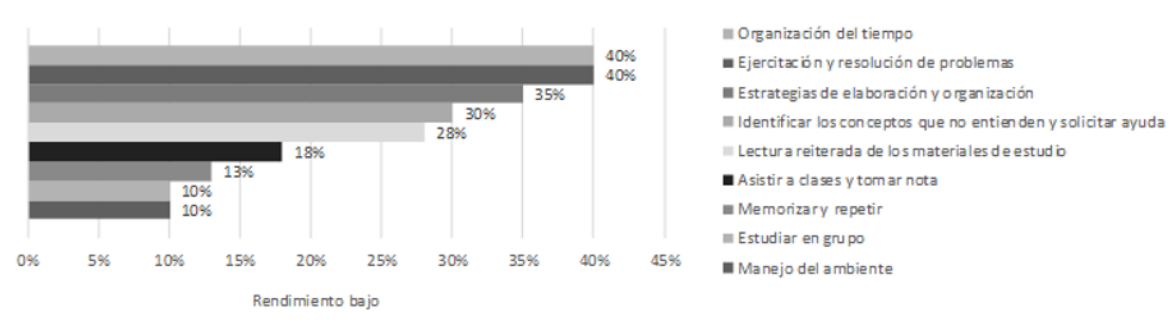

\section{GRÁFICO 2}

Acerca del modo en que se preparan estudiantes con trayectorias de rendimiento bajo para rendir un examen $(\mathrm{N}=40)$ Fuente: Elaboración propia.

Organización del tiempo (40\%). Un 40\% del estudiantado de bajo rendimiento refirió a la organización del tiempo previamente a un examen. Así, se mencionan las horas dedicadas al estudio antes del examen y cómo efectúan los cálculos previos del tiempo - por ejemplo, en términos de semanas - , según la cantidad de materiales de estudio. De manera que se observa en los relatos una sobrevaloración del tiempo, es decir, mientras más prolongadas sean las horas de estudio, mayores chances de aprobar, sin importar qué estrategias utilicen en esos momentos de estudio. Aparece en este grupo de estudiantes la organización del tiempo en relación con otras actividades, como las laborales, por ejemplo, que en los otros grupos analizados no se observan. A continuación, se presentan fragmentos que ilustran sus respuestas.

Cada dia después de la universidad me pongo a repasar, 1 o 2 horas, lo visto desde el comienzo de la materia (S. R., comunicación personal, octubre 2018).

Organizo bien los tiempos de estudio y los tiempos de trabajo (L. S., comunicación personal, agosto 2018).

Ejercitación y resolución de problemas (40\%). También un $40 \%$ del grupo de estudiantes, como parte de su preparación antes de un examen, ejecuta diversos ejercicios y resuelve múltiples problemas, del estilo de los que proponen las y los profesores en clase, los que les proporcionan en sus cuadernillos de estudio e incluso recurren a páginas web.

Inicio realizando una comprensión. Luego lo anoto y hago apuntes. Una semana antes comienzo con la memorización. En caso de que sea práctico, todos los dias hago ejercicios (I. H., comunicación personal, agosto 2018).

Estrategias de elaboración y organización (35\%). Aunque en menor porcentaje que estudiantes de alto rendimiento, aproximadamente un tercio del alumnado de bajo rendimiento mencionó apelar al uso de estrategias como la elaboración de resúmenes, cuadros, esquemas y todos aquellos recursos que les permitan mayor comprensión de conceptos, teorías, relaciones.

Primero subrayo ideas principales y conceptos más importantes del tema, también realizo cuadros sinópticos ( $V$. P., comunicación personal, octubre 2018).

Identificar lo que no comprenden y solicitar ayuda (30\%). Aproximadamente un tercio del estudiantado de bajo rendimiento (la mitad en comparación con los de alto rendimiento) manifestaron que al prepararse para un examen procuran identificar lo que no comprenden para poder solicitar ayuda, ya sea a sus compañeros(as) o a sus docentes.

Realizaba apuntes en clase y pedía ayuda a un/a alumno/a ayudante si habia algo en lo que tuviera dudas (T. R., comunicación personal, octubre 2018).

Lecturas reiteradas de los materiales de estudio (28\%). Casi un tercio del grupo de bajo rendimiento recurre a lecturas repetidas de los materiales, hasta entender, hasta aprenderlo, hasta lograr explicarlos con sus propias palabras. Marcan ideas principales y relacionan algunos conceptos en estas lecturas en profundidad. 
Sin embargo, estas estrategias les permiten un procesamiento menos profundo que las de elaboración u organización de la información.

$\left.1^{\circ}\right)$ Lectura global del contenido, $2^{\circ}$ ) Lectura de los temas que más reconozco $3^{\circ}$ ) Lectura detallada del contenido $\left.4^{\circ}\right)$ Lectura detallada de los temas que menos reconozco (A. Q., comunicación personal, agosto 2018).

Asistir a clases y tomar notas (18\%). Una parte del estudiantado también destacó la importancia de asistir a clases y tomar de notas, no solo porque facilitan la lectura de los materiales de estudio y la resolución de problemas, sino también porque pueden obtener información acerca de cuáles son los contenidos más importantes y los criterios de evaluación.

Mientras se dictaban las clases anotaba todos los detalles o cosas que decía el profesor, las cuales me parecian necesarias (M. D., comunicación personal, octubre 2018).

Memorizar y repetir (13\%). Aunque en un porcentaje bajo (13\%) hizo alusión a estudiar repetitivamente o de memoria.

Primero leo el contenido, trato de entender oración tras oración, subrayo, paso lo subrayado a escrito al cuaderno. Una vez que entiendo lo que me quieren decir los apuntes (el contenido del material a estudiar), lo trato de estudiar de memoria. Una vez que termino de estudiarlo trato de comprender nuevamente el contenido original \#y no el subrayado leyéndolo\# y asi termino (G. G., comunicación personal, septiembre 2018).

Estudio en grupo (10\%). Dentro del grupo de estudiantes de bajo rendimiento, un $10 \%$ hizo referencia al estudio con otros compañeros. Nótese que la misma estrategia fue mencionada con mayor frecuencia por estudiantes de alto rendimiento (28\%).

Nos juntábamos 3 compañeros y realizábamos un resumen, luego nos tomábamos unos días para sabernos bien lo teórico (J. S., comunicación personal, septiembre 2018).

Manejo del ambiente (7\%). Esta categoría involucra estudiantes que destacaron el lugar o ambiente de estudio, como la biblioteca de la universidad, una habitación silenciosa y tranquila, sin molestias externas.

Intentaba ir a la biblioteca a estudiar porque en mi casa no puedo concentrarme (D. M., comunicación personal, agosto 2018).

En resumen, se puede apreciar claramente que aquel estudiantado con trayectoria de rendimiento alto considera importantes, en el momento de prepararse para un examen, las estrategias de elaboración $y$ organización de la información, seguidas por la ejercitación y la resolución de problemas; así como también asistir siempre a clases y ser bueno tomando notas, identificar lo que no comprenden y solicitar ayuda a las y los docentes $o$ a sus compañeros.

En cambio, en el grupo de estudiantes con trayectorias de bajo rendimiento, sobresale la organización del tiempo, fundamentalmente en dos aspectos, por un lado, la prolongación de las horas dedicadas al estudio (sin reflexionar en las estrategias que se utilizan) y por el otro, en la combinación de los momentos de estudios con otras actividades como las laborales. También se destaca la ejercitación y la resolución de problemas como parte fundamental antes de rendir un examen y luego, aunque en menor medida, son consideradas las estrategias de elaboración y organización y aquellas que refieren a identificar lo que no comprenden y solicitar ayuda. Otra de las categorías que surge en este grupo (y que no se presenta entre estudiantes de alto rendimiento) es la lectura reiterada de los materiales de estudio, esto es, leer una y otra vez los materiales hasta comprenderlos. Quizás tiene que ver con una estrategia de repaso que apela a una memorización de conceptos o a lecturas que no demandan mayor actividad a cada estudiante, a diferencia de lo que sucede con la elaboración de un resumen o un cuadro.

Otro señalamiento importante son las discrepancias que se observan entre el análisis cuantitativo del cuestionario y el análisis cualitativo de estas preguntas, especialmente en relación con la estrategia de organización de la información. Si bien el análisis de los ítems no mostró contrastes entre los grupos, el análisis 
cualitativo, da cuenta de que sí existen diferencias en la manera en que abordan los materiales de estudio. En este sentido, el $78 \%$ de estudiantes con trayectorias de alto rendimiento, manifestaron hacer uso de estrategias más comprensivas y analíticas, como lo son las estrategias de organización y elaboración de la información. Mientras, en estudiantes con trayectorias de bajo rendimiento, solo el 35\% expresó que empleaba este tipo de estrategias.

\section{¿Cambió su forma de estudiar desde que entró a la universidad?}

Finalmente, luego de indagar en profundidad cómo estudia el alumnado ingresante, se les preguntó si habían cambiado sus modos de estudiar desde el inicio a la vida universitaria. El objetivo era conocer si a lo largo de su trayectoria en la carrera, habían desarrollado modificaciones en relación con sus hábitos de estudio. Las alternativas de respuesta eran sí y no. En el caso que la respuesta fuera positiva, debían describir qué aspectos habían decidido modificar en cuanto a las maneras de estudiar.

El Gráfico 3 sintetiza los resultados obtenidos:

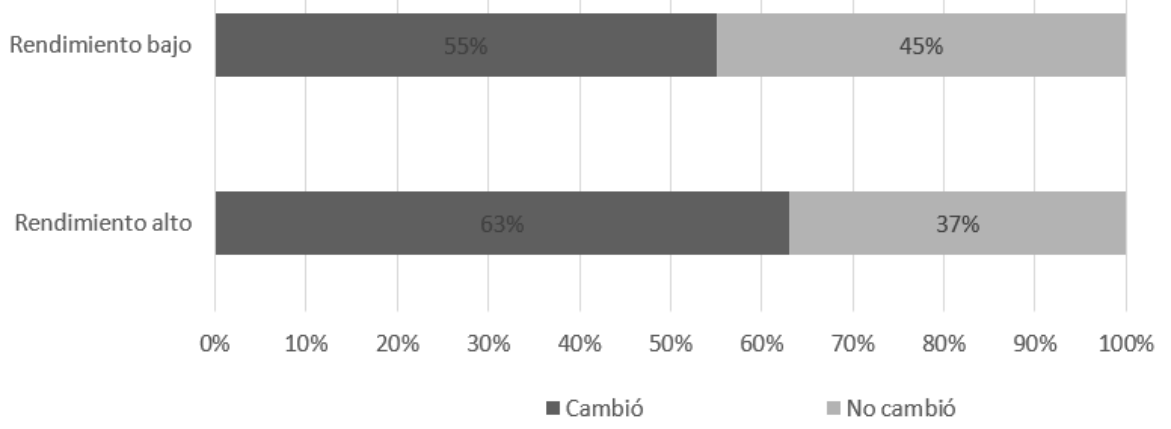

GRÁFICO 3

Cambios en los hábitos de estudio desde el ingreso a la Universidad, según trayectoria de cursado $(\mathrm{N}=86)$ Fuente: Elaboración propia

En los dos grupos analizados un importante porcentaje reconoció haber desarrollado modificaciones en los modos de estudiar o prepararse ante un examen, siendo ligeramente superior en estudiantes con trayectorias de rendimiento alto. Sin embargo, al consultarles específicamente qué aspectos habían logrado modificar, no todo el estudiantado logró explicitarlos de manera detallada. En efecto, dentro del grupo de estudiantes de bajo rendimiento, solo el $50 \%$ de quienes reconocieron haber cambiado sus hábitos de estudio, especificaron con claridad aquello que habían modificado, el resto de estudiantes de este grupo no respondió a este ítem.

Todo el alumnado que respondió a esta pregunta hizo referencia al tiempo dedicado y los materiales de estudio. Sin embargo, no tienen el mismo significado en los dos grupos. En el caso de estudiantes con trayectorias de rendimiento alto, la cuestión del tiempo aparece vinculada a su gestión, es decir, a la planificación, a la organización y a la búsqueda de las estrategias más efectivas para cumplir las metas de aprendizaje (55\%). Y en segundo lugar (38\%) emerge la categoría relacionada con la manera en que estos estudiantes consideran que deben enfrentarse a los materiales de estudio: buscando una comprensión profunda de estos, estableciendo relaciones, integrando otras fuentes de información, analizando críticamente lo que aprende.

De manera contraria, los cambios que manifestó el alumnado de rendimiento bajo en relación con el tiempo, tienen que ver con el aumento en las horas de dedicación (82\%) lo que no siempre garantiza el alcance de buenos resultados si no se reflexiona sobre las estrategias de estudio que permitan el abordaje más profundo de los materiales. 
Un señalamiento importante de este grupo es que de todos los que respondieron afirmativamente haber cambiado sus estrategias de estudio al largo de su experiencia en la universidad, solo una parte pudo explicar con precisión qué aspectos modificaron - el 50\%, el resto, no respondió a este ítem.

Esto indica de alguna manera cuán importante es el desarrollo de habilidades metacognitivas, no solo para regular los propios procesos de aprendizaje, sino también para mejorarlos, impactando de manera positiva en el desempeño académico.

\section{Conclusiones}

El objetivo de este trabajo fue describir las estrategias de aprendizaje empleadas por los ingresantes universitarios durante su experiencia en los primeros meses en las carreras de ingeniería, con la finalidad de analizar sus implicancias en las trayectorias de logro y de bajo rendimiento académico.

Se analizaron trayectorias de 86 estudiantes de los cuales el 53\% ( $\mathrm{N}=46)$ correspondía al grupo de estudiantes con trayectorias de rendimiento alto, esto es, habían logrado regularizar todas las asignaturas correspondientes al primer cuatrimestre y el $47 \%(\mathrm{~N}=40)$ restante correspondía al grupo con trayectorias de rendimiento bajo, puesto que no habían regularizado ninguna de las asignaturas cursadas.

Los resultados obtenidos a partir de la administración del cuestionario mostraron algunas diferencias interesantes, que permitirían dilucidar relaciones positivas entre el uso de algunas estrategias cognitivas complejas (como la elaboración de la información), estrategias metacognitivas (identificar lo que no comprenden, planificación, control y evaluación) y de manejo de recursos (como la regulación del esfuerzo, la organización del tiempo y la disposición para solicitar ayuda a las y los profesores) y el buen desempeño académico.

Cuando se profundiza en esos datos a través de las respuestas obtenidas a las preguntas abiertas formuladas en el cuestionario se marcaron también diferencias entre los grupos de alto y bajo rendimiento.

¿Cómo se preparan para rendir un examen? Estudiantes con trayectorias de rendimiento alto, cuando estudian, prefieren optar por estrategias cognitivas de elaboración y organización que les permitan un procesamiento más complejo o profundo de los materiales de estudio, a través del establecimiento de relaciones y la construcción de nuevos significados a partir de lo ya aprendido. Consideran necesario como parte de sus hábitos de estudio resolver ejercicios y situaciones problemáticas. Resulta interesante que además de las estrategias de aprendizaje mencionadas, asistir a clases y ser buenos tomando nota, es algo que este grupo considera indispensable para el logro académico, al igual que identificar lo que no comprenden para solicitar ayuda.

En cuanto estudiantes con trayectorias de rendimiento bajo, cuando se les preguntó cómo se preparaban para rendir un examen, la categoría que emerge en primer lugar es la organización del tiempo, en el sentido de la cantidad de horas que dedican al estudio, sin reflexionar acerca de cuáles son las estrategias que utilizan. Asimismo, aparece la dimensión temporal en la combinación del estudio con otras actividades, como las laborales, por ejemplo, que en el otro grupo no aparece. También destacan la ejercitación y la resolución de problemas y en menor medida son consideradas las estrategias de elaboración y organización y aquellas que refieren a identificar lo que no comprenden y solicitar ayuda. Otra de las categorías que surge como característica de este grupo, es la lectura reiterada, la cual podría asociarse a las estrategias de repaso y estudiar de memoria.

Finalmente, al preguntarles si habían cambiado sus formas de estudiar desde que ingresaron a la universidad, no hubo diferencias notorias entre los dos grupos de estudiantes. De hecho, en ambos grupos, más del 50\% señaló haber realizado algún tipo de modificación en sus hábitos de estudio. Sin embargo, no todo el alumnado pudo especificar qué cambios desarrollaron; particularmente entre estudiantes de rendimiento bajo, solamente la mitad especificó qué era lo que había modificado en sus hábitos de estudio mientras que la otra mitad no respondió. 
De manera general, se podría decir que todos los que respondieron a esta pregunta hicieron referencia al tiempo dedicado al estudio, sin embargo, para de rendimiento alto, el tiempo aparece vinculado a su gestión, es decir, a la planificación, a la organización y a la búsqueda de las estrategias más efectivas para cumplir las metas de aprendizaje (55\%). A diferencia de ello, los cambios que manifestaron estudiantes de rendimiento bajo en relación con el tiempo, tienen que ver con el aumento en las horas de dedicación (82\%) lo que no siempre garantiza el alcance de buenos resultados si no se reflexiona acerca de las estrategias que permitan el abordaje más profundo de los materiales de estudio.

Los resultados obtenidos plantean la importancia de conocer las estrategias que el alumnado emplea cuando se enfrentan a situaciones de aprendizaje y de diseñar e implementar instancias formativas al respecto. De hecho, si se conoce que determinados modos de afrontar el estudio y el aprendizaje son más efectivos y suelen asociarse más frecuentemente con mejores rendimientos, se dispone de los insumos para diseñar talleres, cursos, instancias de participación optativa que desde el ingreso mismo apuntalen al recién llegado a la universidad.

Año tras año, ingresa en la universidad una población estudiantil con formación académica previa variada, con hábitos y estrategias heterogéneas. Reconocer sus fortalezas y debilidades, no solo permite identificar los factores que pueden llegar a afectar su desempeño académico y efectuar propuestas para mejorar sus formas de aprender, sino que también proporcionarían pistas que permitirían desarrollar las herramientas para acompañar y fortalecer sus trayectorias académicas desde el inicio a la vida universitaria (Garzuzi, 2015).

Si bien es cierto que los aprendizajes en la universidad requieren del manejo de determinadas habilidades, estrategias y conocimientos, existen destrezas que son específicas de las disciplinas elegidas y necesariamente se aprenden con la experiencia. Es decir que el hecho de atravesar diversas situaciones de aprendizaje y formar parte de ellas, permite el desarrollo de ciertas habilidades autorregulatorias del aprendizaje. No obstante el énfasis en el inicio de los estudios universitarios debería estar puesto en el desarrollo de intervenciones primarias que incrementen el nivel de compresión y conciencia que tienen las y los estudiantes respecto de sus retos académicos y sociales del nuevo entorno y a la vez, proporcionar estímulos para el desarrollo del control interno en los modos de aprender, a tener cierta evaluación crítica del conocimiento transmitido y a reforzar habilidades de meta estudio (García, et al., 2016).

A modo de cierre y en palabras de Garzuzi (2015) resulta interesante pensar a la universidad en un rol orientador que desarrolle planes de acción para el acompañamiento de estudiantes en el desarrollo de estrategias que contribuyan a un aprendizaje autorregulado, sin ignorar que no son independientes de los contextos donde se ponen en práctica y la incidencia de otros factores, tales como los familiares, las historias de vida, la formación previa del nivel medio, las instituciones, factores motivacionales, entre muchos otros.

\section{REFERENCIAS}

Aparicio, M. (2009). La demora en los estudios universitarios: causas desde una perspectiva cuantitativa. EDIUNC: Mendoza.

Bossolasco, M. L., Chiecher A. y Dos Santos, D. A. (2019). Diseño y validación de un instrumento para el análisis de trayectorias académicas en el primer año universitario. Revista Educación Superior. 18(27), 11-38. Recuperado de http://revistavipi.uapa.edu.do/index.php/edusup/article/view/180/pdf

Broc C. M. (2011). Voluntad para estudiar, regulación del esfuerzo, gestión eficaz del tiempo y rendimiento académico en alumnos universitarios. Revista de Investigación Educativa. 29(1), 171-185. Recuperado de https://core.ac. uk/download/pdf/41554638.pdf

Chiecher, A., Paoloni, P. y Guebara, J. (2011). Abandonadores de las carreras de ingeniería. Motivo de abandono de los estudios y definición de nuevas metas. Argentina: Universidad Nacional de Río Cuarto. Recuperado de https:// bit.ly/2VQNSp5 
Donolo, D., Chiecher, A., Paoloni P. y Rinaudo, C. (2008). MSLQ. Propuestas para la medición de la motivación y el uso de estrategias de aprendizaje. Río Cuarto: Editorial de la Universidad Nacional de Río Cuarto.

García, A. (2015). Políticas institucionales para mejorar la retención y la graduación en las Universidades Nacionales Argentinas. Revista Debate Universitario, 7(4), 7-24. Recuperado de http://ppct.caicyt.gov.ar/index.php/debat euniversitario/article/view/7641/pdf

García, J. C., Gonzáles, M. y Zanfrillo, A. (2011). Desgranamiento universitario: perspectiva estudiantil en ingeniería. En XI Coloquio Internacional de Gestión Universitaria de América del Sur. Universidad de Santa Catarina, Florianópolis, Brasil.

García, R. M., Sánchez G. M. y Risquez, A. (2016). Estrategias de Aprendizaje y Autorregulación Motivacional. Identificación de Perfiles para la Orientación de Estudiantes Universitarios de Nuevo Ingreso. Revista Iberoamericana de Diagnóstico y Evaluación, 41(1), 39-57. Recuperado de https://www.redalyc.org/pdf/4596/ 459646901005.pdf

Garello, M. y Rinaudo, M. (2012). Características de las tareas académicas que favorecen aprendizaje autorregulado y cognición distribuida en estudiantes universitarios. RedU Revista de Docencia Universitaria, 10, 415 - 440.

Garzuzi, V. (2015). El desarrollo de estrategias de aprendizaje en la trayectoria académica universitaria. En I. L. Morchio (Coord.) Aprender a aprender como meta de la Educación Superior: desde la comprensión de cómo aprende el alumno universitario a la promoción del aprendizaje autorregulado. (pp. 171-201). Buenos Aires: Teseo.

Hernández R., Fernández, C. y Baptista, P. (2014). Metodología de la Investigación (6ta ed.). México: Mc Graw Hill.

Moreno, J. E y Chiecher, A. (2014). Redes sociales en espacios educativos. Una propuesta para favorecer el ingreso universitario. Revista Iberoamericana de Educación en Tecnología y Tecnología en Educación. (14), 55-64. Recuperado de https://teyet-revista.info.unlp.edu.ar/TEyET/article/view/324

Panaia, M. (2013). Abandonar la universidad ¿Decisión premeditada o imprevista? En M. Panaia (Coord.) Abandonar la Universidad con o sin título. Buenos Aires: Miño y Dávila.

Parrino, M. C. (2010). Deserción en el primer año universitario. Dificultades y logros. En X Coloquio Internacional sobre Gestión Universitaria en América el Sur. Mar del Plata.

Parrino, M. C. (2014). Factores intervinientes en la deserción universitaria. Revista Argentina de Educación Superior, 6(8), 39-61. Recuperado de https://dialnet.unirioja.es/servlet/articulo?codigo $=4753784$

Pintrich, P. y García, T. (1993). Intraindividual diffrences in students' motivation and selfregulated learning. German Journal of Educational Psichology, 7(3), 99-107.

Pintrich, P., Smith, D. García T. y McKeachie W. (1991). A manual for the use of the Motivated Strategies for Learning Questionnaire (MSLQ). Michigan: National Center for Research to Improve Postsecondary Teaching and Learning.

Pozo, J. I. (1990). Estrategias de aprendizaje. En C. Coll, J. Palacios y A. Marchesi (Comp). Desarrollo psicológico y educación. Psicología de la Educación (pp 199-221). Madrid: Alianza Editorial.

Sierra, H. y Hernández O. (2016). Sistema de alertas tempranas como herramienta de innovación tecnológica en la Universidad Santo Tomás para el fortalecimiento de la permanencia estudiantil y graduación oportuna. Ponencia llevada a cabo en VI Congresos CLABES. Medellín, Colombia. Recuperado de https://revistas.utp.ac.pa/index .php/clabes/article/view/1056

Terigi, F. (2009). Las trayectorias escolares. Del problema individual al desafio de politica educativa. Argentina: Ministerio de Educación. Recuperado de http://www.bnm.me.gov.ar/gigal/documentos/EL004307.pdf

Vera S. A., Poblete, C. S. y Díaz, L. C. (2019). Percepción de estrategias y estilos de aprendizaje en estudiantes universitarios de primer año. Revista Cubana de Educación Superior. (38), 1-23. Recuperado de http://www.rc es.uh.cu/index.php/RCES/article/view/276/318

\section{Notas}

[1] Ingeniería Mecánica, Ingeniería Química, Ingeniería Electricista e Ingeniería en Telecomunicaciones. 
[2] Se puede apreciar cómo a medida que baja el rendimiento, disminuyen los porcentajes de estudiantes que efectivamente dieron respuesta al instrumento de recolección de datos administrado. En efecto, el trabajo de campo fue arduo, y más todavía el contacto con estudiantes de bajo rendimiento, muchos de los cuales habían abandonado la carrera.

[3] Enlace para acceder al cuestionario: https://goo.gl/forms/cDPARFz8iUWMnxtG3

[4] El instrumento fue sometido a un procedimiento de validez aparente y de contenido, mediante la técnica de juicio de expertos. Los resultados del procedimiento, para la sección de estrategias de aprendizaje, arrojaron altas valuaciones de los jueces para todos los ítems que la componen ( $\mathrm{M}=3,83$ a interpretarse en una escala de 4 puntos, en la que el valor máximo indica alta pertinencia de los ítems para medir el aspecto o variable referida). Asimismo, las altas valuaciones estuvieron acompañadas por altos índices de concordancia (Bossolasco, Chiecher y Dos Santos, 2019).

[5] Se trata de categorías no mutuamente excluyentes.

\section{BY-NC-ND}

